\title{
A new insight for the screening of potential $\beta$-lactamase inhibitors
}

\author{
Vijai Singh $^{1 *}$, Dharmendra Kumar Chaudhary ${ }^{2}$ \\ 1 Department of Biotechnology, Invertis University, Bareilly- Lucknow National Highway-24, Bareilly 243123, India \\ 2 Department of Zoology and Biotechnology, H.N.B. Garhwal Central University, Campus Badshahithaul 249199, Tehri \\ Garhwal Uttarakhand, India
}

* Corresponding author: vijaisingh15@gmail.com

\begin{abstract}
The $\beta$-lactamase produces by Aeromonas hydrophila which enables to hydrolyze and inactivate $\beta$ lactam ring of antibiotics. The homology modeling was used to generate the 3-D model of $\beta$-lactamase by using known template 3-D structure. The stereochemical quality and torsion angle of 3-D model were validated. Total eleven effective drugs have been selected and targeted the active amino acid residues in $\beta$-lactamase. The drugs were derivative of $\beta$-lactam ring antibiotics and screening was made by docking. Out of 11 drugs, 3 drugs (Ampicillin, Astreonam and Sultamicillin) were found to be more potent on the basis of robust binding energy between protein-drug interactions. Additionally, homology of $\beta$-lactamase of $\boldsymbol{A}$. hydrophila resembled with other pathogenic bacteria that used for phylogeny analysis. These findings suggest a new insight for better understanding and useful for designing of novel potent drugs.
\end{abstract}

Keywords: Aeromonas hydrophila; $\beta$-lactamase; homology modeling; drugs; docking

\section{INTRODUCTION}

$A_{\mathrm{p}}^{\text {E }}$ EROMONAS hydrophila is an opportunistic bacterial pathogen which causes hemorrhagic septicemia in fish, reptiles, amphibians (Austin and Austin 1999) and soft tissues infection and diarrhea in human (Barghouthi et al., 1989). There are fifty cases of Aeromonas septicaemia reported in severe hepatic cirrhosis and found $52 \%$ A. hydrophila isolates. A case of liver cirrhosis with $A$. hydrophila infection presenting as acute gastroenteritis and non-traumatic acute osteomyelitis. It has been shown that $A$. hydrophila frequently affects immunocompromised patient with liver cirrhosis (Lee et al., 2003). A. hydrophila secretes a number of extracellular enzymes including proteases, DNase, RNase, elastase, lecithinase, amylase, lipase, gelatinase, chitinase (Merino et al., 1995; Pemberton et al., 1997) and cytotoxic/ cytolytic enterotoxins (Chopra et al., 1993) and three haemolysins (Hanes and Chandler 1993; Hirono and Aoki 1991; Howard and Buckley 1985). There is a pressing need to diagnose and cure $A$. hydrophila infections.

A. hydrophila have been studied for antibiotic sensitivity assay and found all isolates (total no. 25) were resistant to Cephalothin, Ampicillin, Novobiocin and Nitrofurazone, and sensitive to Gentamicin (80\%), Co-trimaxazole (92\%), Chloramphenicol and Ciprofloxacin (Rathore et al., 2006). An urgent need arises to develop vaccine for controlling of infection but not available yet. Therefore, antibiotics are an alternative and widely used for controlling of $A$. hydrophila infection in human and other animals. $\beta$-lactamase is one of the most significant enzymes produces from $A$. hydrophila. The $\beta$-Lactam antibiotics have been used for controlling of microbial infections. Bacteria have been evolved to hydrolyze the $\beta$-lactams by production of $\beta$-lactamases (Frere 1995). There is heterogeneity of $\beta$-lactamases thus, inhibitors are ineffective. However, some inhibitors are effective only against serine $\beta$-lactamases as they are hydrolyzed by metallo $\beta$-lactamases (MBLs). A number of inhibitors for MBLs have been reported their side chains binding in a predominantly hydrophobic pocket while their functional groups interacted with zinc ions (Heinz et al., 2003; Siemann et al., 2002).

The 3-D model of $\beta$-lactamase of $A$. hydrophila is unknown so for. The homology modeling is used to generate the 3-D model of $\beta$-lactamase by using the known 3-D crystal structure as a template. While molecular docking is used for screening of potent and specific drugs by targeting active amino acid residue in $\beta$-lactamase. There are a number of previous reports for used of homology modelling of $\beta$ ketoacyl acyl carrier protein synthase (KAS) III of Enterococcus faecalis. Similarly, homology modeling has been used to generate the 3D model of KASIII protein. The identification of active site residue has been performed using docking and found the two antibacterial drugs for inhibition of growth (Jeong et al., 2007). In this study, homology modeling has also been used for construction of 3-D model of NAD ${ }^{+}$dependent DNA ligase of Mycobacterium tuberculosis. The screening of a number of drugs has been performed by docking approach (Srivastava et al., 2005). The phylogenetic relationship based of protein homology is vital for better understanding of genetic evolutionary relationship of organisms. The aim of present study is to generate the 3-D model of $\beta$-lactamase and screening of potent drugs.

\section{MATERIALS AND METHODS}

\subsection{Retrieval and searching of sequences}

The complete protein sequences of $\beta$-lactamase from $A$. hydrophila and other bacteria strains were retrieved from National Centre for Biotechnology Information (http://www.ncbi.nlm.nih.gov). The relatedness of sequences 
deposited in databases was evaluated by BLAST (Basic Local Alignment Search Tool) (Altschul et al., 1990) that was implemented via NCBI (http://www.ncbi.nlm.nih.gov/blast). The BlastP (protein query-protein database comparison) was performed with protein data bank (PDB). The alignment was also performed with target protein sequences with template protein (PDB: 1X8G) using CLUSTAL X 1.83.

\subsection{Generation and evaluation of 3-D model}

The X-crystal structural mono zinc carbapenemase (Cpha) from $A$. hydrophila was available at $1.70 \AA$ resolution (PDB: $1 \mathrm{X8G}$ ) that was used as template structure to generate 3-D model of $\beta$-lactamase. The homology modeling was used to generate the 3D structure of $\beta$-lactamase through the Modeller9v2. It was evaluated by minimum model score and dope score of model and template. The 3D model was further validated by PROCHECK that also created a Ramachandran plot. The accuracy and overall G- factor were also considered for model evaluation.

\subsection{Screening of potent drugs}

The drugs were taken from NCBI Pubchem compounds in 2-D structure that was converted into 3-D structure using Babel tool. The 3-D model of $\beta$-lactamase and drug were used for docking. A number of drugs were docked using AutoDock against $\beta$-lactamase model. The Lamarckian Genetic Algorithm (LGA) of the Autodock 3.05 was used for docking experiments. Distance-dependent function of the dielectric constant was used for the calculation of the energetic maps and all other parameters were used by default value.

\subsection{Construction and analysis of phylogenetic tree}

The protein sequence of $\beta$-lactamase of $A$. hydrophila was used for searching the homology with BLAST and homologous sequences were retrieved Genbank. All these protein sequences were aligned in CLUSTALX 2.0.5. These sequences used passion correction equation which was implemented in the MEGA 4.0 program for construction of a phylogenetic tree by neighbor-joining method. A total of 1000 bootstrapped values were sampled to determine a measure of the support for each node on the consensus tree.

\section{RESULTS AND DISCUSSION}

The size of $\beta$-lactamase (NCBI Accession No. ABK35804.1) of A. hydrophila subsp hydrophila ATCC 7966 was 253 amino acids long that showed the structural homology with 3-D crystal structure (PDB: $1 \times 8 \mathrm{~g}$ ) of mono zinc carbapenemase (cphA) of $A$. hydrophila. The percentage identity of $\beta$ lactamase with template was $86 \%$ and positive amino acid residue was $88 \%$. E-value score showed in the BLAST result for selected 3-D structure was 1e-130. In the present study, we observed significant similarity of score and dope score target and template protein. Total five models were generated and selected the one 3-D model $\beta$ - lactamase which showed similar free energy. The 3-D model of $\beta$ - lactamase of $A$. hydrophila was shown (Fig. 1). It contains the three major lobe first (red) lobe $2 \alpha$-helix and $3 \alpha$-helix in green lobe whereas the $9 \beta$-sheets.

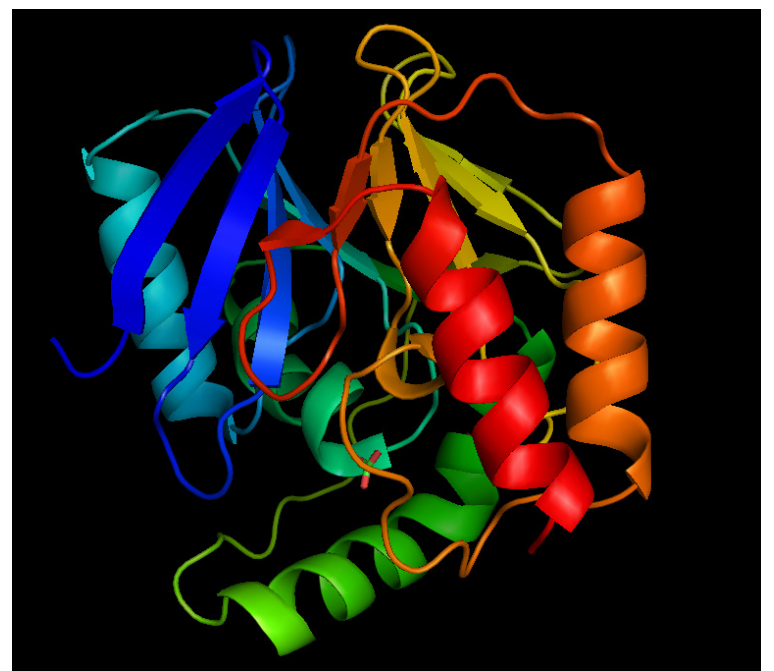

Fig.1 The 3-D model of $\beta$-lactamase of $A$. hydrophila.

The similar approach has been previously applied for cry2Abtype gene of Bacillus thuringiensis. The protein model was constructed by homology modeling and predicted a receptorbinding site by docking (Lin et al., 2008). The 3-D models for the $65-\mathrm{kDa}$ of Cry4A and Cry4B endotoxins of $B$. thuringiensis has been constructed by homology modeling based on crystal structure of Cry1Aa and Cry3Aa (Angsuthanasombat et al., 2004). Whereas stereochemical and spatial arrangement of amino acids residues within the most favored region in the Ramachandran plat is determined. The torsion angle of 3-D model of $\beta$-lactamase of $A$. hydrophila was $91.1 \%$ in favored region whereas $0.9 \%$ amino acid residue in disallowed region. The overall G-factor of 3-D model was $1.0 \%$ as it indicated the best protein model.

In a previous study, homology modeling has been used for generating a 3-D model of $\beta$-ketoacyl acyl carrier synthase (KAS) III protein of Enterococcus faecalis. The generation of 3$D$ model has been done by through Modeller that was validated by using PROCHECK programme. In this study, the torsion angle of $88.9 \%$ of residues had within the allowed region and only $0.3 \%$ of residues in disallowed region of the Ramachandran plot. Similar approach has been applied in another study, identification of active site residue using docking and found Naringenin and Apigenin that was able to inhibit the growth of bacteria in culture (Jeong et al., 2007). The $\beta$-lactamase is the one of most known enzymes that degrades $\beta$-lactam ring. In the present study, total 11 drugs were selected for docking with active amino acid residues of $\beta$-lactamase. Out of 11 drugs, 3 drugs (Ampicillin, Astreonam and Sultamicillin) were found to be highest binding energy means lowest docked energy (Table 1). The docking energy of Ampicillin, Astreonam and Sultamicillin were -20.80, 21.05 and -20.88 respectively. On the other hand, these drugs have relatively similar interaction energy with protein. 
bioRxiv preprint doi: https://doi.org/10.1101/005181; this version posted May 15,2014 . The copyright holder for this preprint (which was not certified by peer review) is the author/funder, who has granted bioRxiv a license to display the preprint in perpetuity. It is made available under aCC-BY 4.0 International license.

There is a hydrogen bond (HB) observed that can be helpful for stronger binding between protein and drug molecules. The active amino acid residues of $\beta$-lactamase showed the affinity with Ampicillin, Astreonam and Sultamicillin were given (Fig. 2, 3, 4).

Table 1 The interaction energy $\left(\mathrm{kcal}^{\mathrm{mol}} \mathrm{m}^{-1}\right.$ ) of $\beta$-lactamase and drugs obtained by docking.

\begin{tabular}{|l|l|l|}
\hline Drugs & $\begin{array}{l}\text { Binding energy } \\
\left(\mathrm{kcal}^{\left.-\mathrm{mol}^{-1}\right)}\right.\end{array}$ & $\begin{array}{l}\text { Docked energy } \\
\left(\mathrm{kcal}^{-\mathrm{mol}^{-1}}\right)\end{array}$ \\
\hline Ampicillin & -18.75 & -20.80 \\
\hline Domicillin & -17.24 & -17.62 \\
\hline Polycillin & -18.39 & -19.45 \\
\hline Astreonam & -23.54 & -21.05 \\
\hline Cephalosporin & -07.23 & -06.70 \\
\hline Penicillin & -10.82 & -13.96 \\
\hline Pivampicillin & -15.62 & -18.15 \\
\hline Sultamicillin & -18.20 & -20.88 \\
\hline Takacillin & -17.94 & -18.12 \\
\hline Capicillin & -11.50 & -14.28 \\
\hline Novocillin & -10.72 & -14.06 \\
\hline
\end{tabular}

The HB was formed between $\beta$-lactamase and Ampicillin in the active amino acid such as Thr97 and Asp98 with 2.15 and 2.25 Á distances (Fig. 2). In other drugs (Astreonam), HB was formed with active amino acids that include Thr97 and Thr34 with the 2.477 and 2.507 $\mathrm{A}$ distances (Fig. 3). While HB was formed between $\beta$-lactamase and Sultamicillin such amino acids Gln48 and Asn44 with1.808 and 2.215 Á distances (Fig. 4).

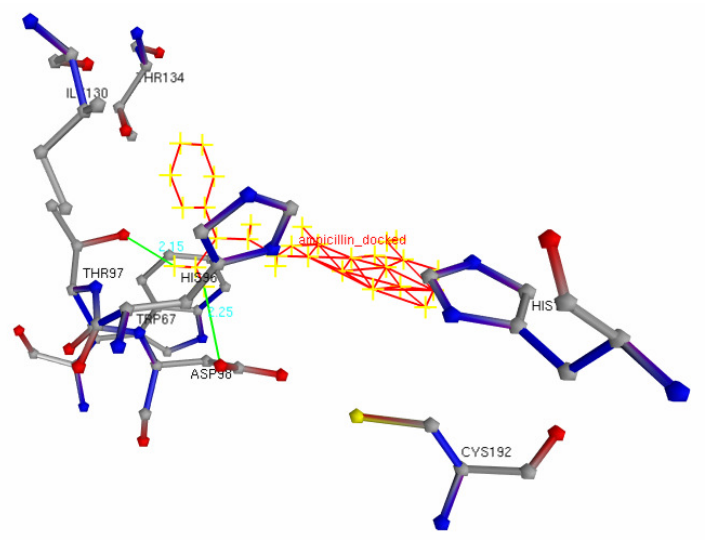

Fig.2 The interaction of high affinity Ampicillin drugs with $\beta$ lactamase.

The phylogenetic tree of $\beta$-lactamase of $A$. hydrophila and other potential bacteria were constructed. Therefore, homology of $\beta$-lactamase was present in a number of bacteria. As depicted in Fig.5, phylogenetic tree of the $\beta$ lactamase of $A$. hydrophila with other bacteria that contains homologous protein. The $\beta$-lactamase of $A$. hydrophila showed the closely related with $A$. sobria, $A$. salmonicida, $A$. caveai, $A$. veronii, $A$. jandaei and $A$. allosaccharophila which was present in the same clade.
The other groups of significant bacteria present in other clades.

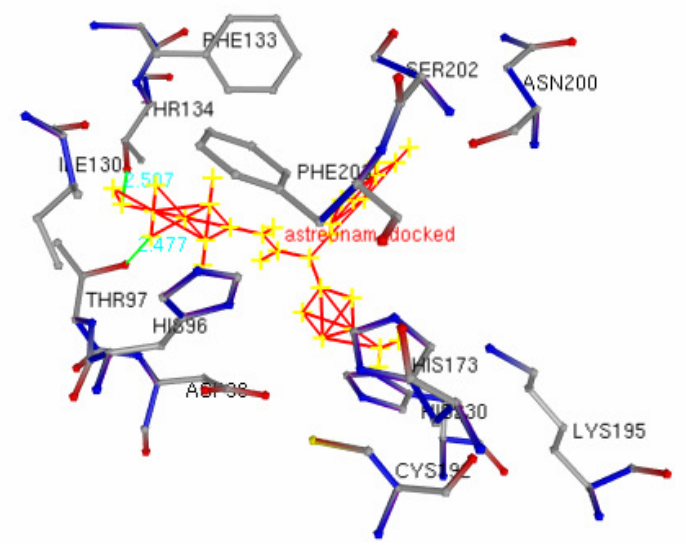

Fig.3 The interaction of high affinity Astreonam drugs with $\beta$ lactamase.

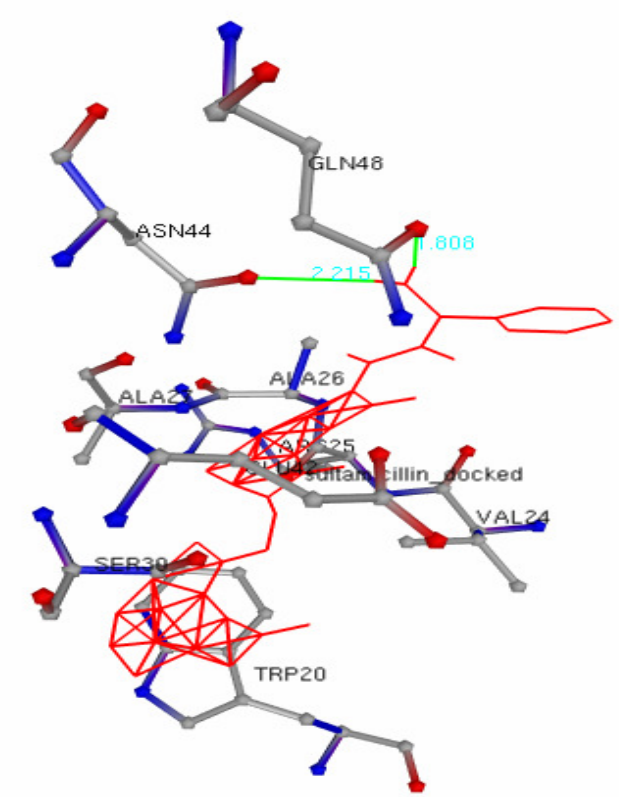

Fig.4 The interaction of high affinity Sultamicillin drugs with $\beta$-lactamase.

However, these bacteria contain homologous region of $\beta$ lactamase. It indicates that Ampicillin, Astreonam and Sultamicillin can also be used for controlling of growth of Aeromonas species and also used for other bacteria. The phylogeny indicates that $\beta$-lactamase is a stable target for genetic relationship. In a previous study, nucleotide sequences of gyrB have been used for confirmation of 53 Aeromonas strains including some new isolates that was characterized by 165 rDNA sequences (Yanez et al., 2003). The phylogeny of Aeromonas bestiarum and Aeromonas salmonicida has been determined in 70 strains using rpoD sequence encoding the sigma70 factor. This analysis was complemented with the sequence of gyrB which had already proven useful for determining the phylogenetic relationship. 


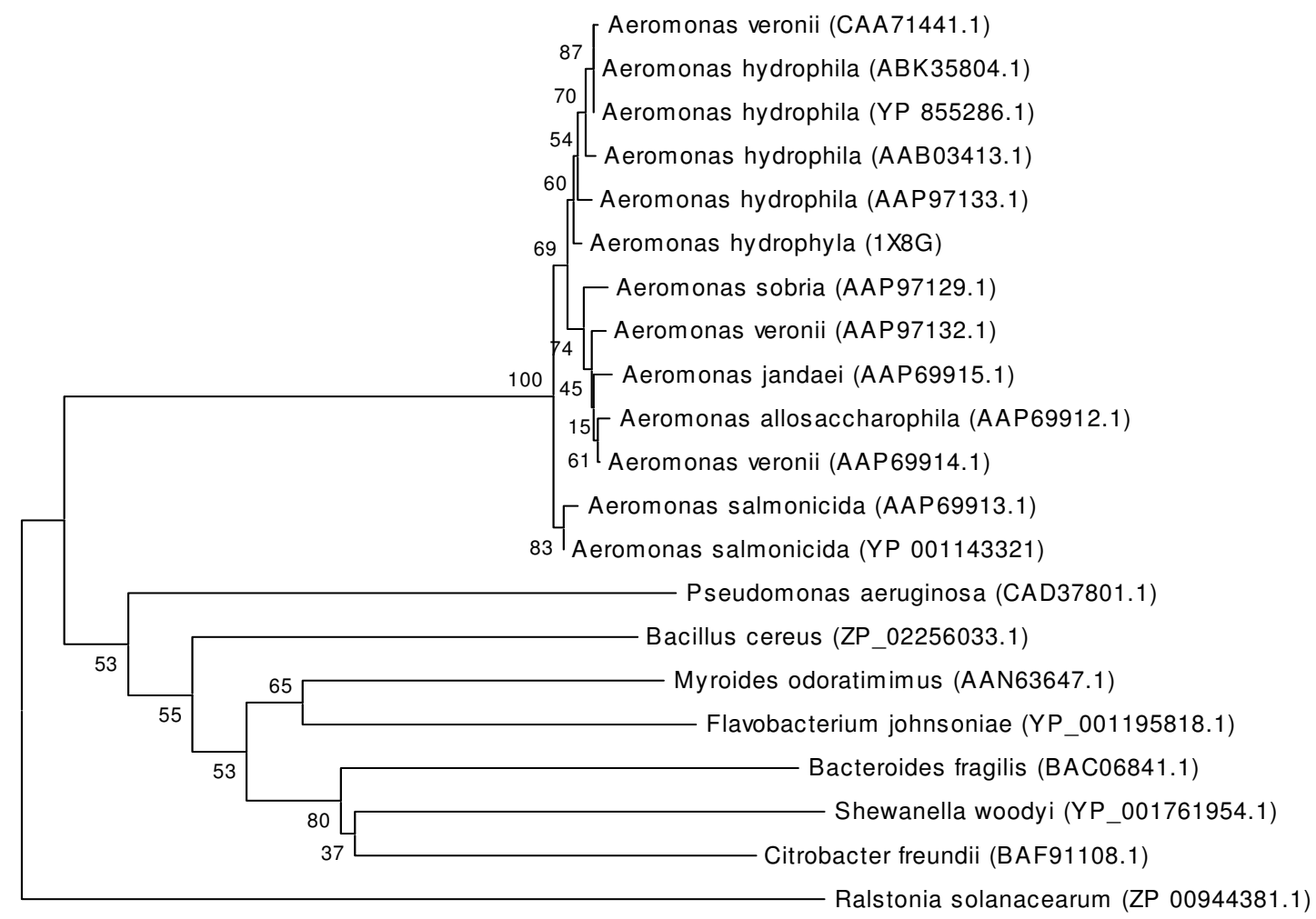

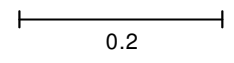

Fig.5 Phylogenetic tree of $\beta$-lactamase of $A$. hydrophila with other significant bacteria. The bar 0.2 represent the changes per site.

\section{CONCLUSION}

We have successfully constructed a 3-D model for $\beta$ lactamase of $A$. hydrophila that could be used for screening of potent drugs. We found that Ampicillin, Astreonam and Sultamicillin have highest binding affinity. It means that stronger inhibition of growth of $A$. hydrophila. Whereas, phylogeny of $\beta$-lactamase of $A$. hydrophila indicates that the same drug can be inhibited the growth of other bacteria. This study also provides a new insight for controlling of unwanted and heavy use of drug in lab practices. The excessive use of antibiotics has accelerated the spreading of drug-resistant strains that may be a grand challenge.

\section{ACKNOWLEDGEMENT}

Authors thank Indra Mani and Satya Prakash for their critical review and suggestion.

\section{REFERENCES}

Altschul S.F., Gish W., Miller W., Myers E.W., and Lipman D.J. (1990). Basic local alignment search tool. J Mol Biol. 215(3):403-10.

Angsuthanasombat C., Uawithya P., Leetachewa S., Pornwiroon W., Ounjai P., Kerdcharoen T., Katzenmeier G. and Panyim S. (2004). Bacillus thuringiensis Cry4A and Cry4B Mosquito-larvicidal proteins: Homology based 3-D model and implications for toxin activity. J. Biochem. Mol. Biol. 37(3):304-313.

Austin B., Austin D.A. (1999). Bacterial fish pathogens: Diseases in farmed and wild fish. Praxis Publishing, Chichester, UK.

Barghouthi S., Young R., Olson M.O., Arceneaux J.E., Clem L.W., Byers B.R. (1989). Amonabactin a noval trypophan or phenylalanine- containing phenolate siderophore in Aeromonas hydrophila. J. Bacteriol. 171:1811-1816.

Chopra A.K., Houston C.W., Peterson J.W., Jin G.F. (1993). Cloning, expression and sequence analysis of a cytolytic enterotoxin gene from Aeromonas hydrophila. Can. J. Microbiol. 39(5):513-523.

Frere, J. M. (1995). $\beta$-Lactamases and bacterial resistance to antibiotics. Mol. Microbiol. 16:385-395.

Hanes D.E., Chandler D.K.F. (1993). The role of a 40 megadalton plasmid in the adherance and hemolytic properties of Aeromonas hydrophila. Microb. Pathog. 15:313-317.

Heinz, U., Bauer R., Wommer S., Meyer-Klaucke W., Papamichaels C., Bateson J., Adolph H.W. (2003). Coordination geometries of metal ions in D- or L-captopril-inhibited metallo-_-lactamases. J. Biol. Chem. 278:20659-20666.

Hirono I., Aoki T. (1991). Nucleotide sequence and expression of an extracellular hemolysin gene of Aeromonas hydrophila'. Microb. Pathog. 11:189-197.

Howard S.P., Buckley J.T. (1985). Activation of the hole forming toxin aerolysin by extracellular processing. J. Bacteriol. 163:336-340.

Jeong K.W., Lee J.Y., Kim Y. (2007). Homology modeling and docking study of beta-ketoacyl acy carrier protein synthase III from Entercoccus faecalis. Bull. Korean Chem. Soc. 28(8):1335-1340.

Lee C.H., Liu M.S., Hsieh S.H. (2003). Aeromonas hydrophila bacteremia presenting as non-traumatic acute osteomyelitis in a cirrhotic patient. Chang Gung Med. 26(7):520-524. 
bioRxiv preprint doi: https://doi.org/10.1101/005181; this version posted May 15, 2014. The copyright holder for this preprint (which was

not certified by peer review) is the author/funder, who has granted bioRxiv a license to display the preprint in perpetuity. It is made available under aCC-BY 4.0 International license.

Lin Y., Fang G., Cai F. (2008). The insecticidal crystal protein Cyr2Ab10 from Bacillus thuringiensis cloning, expression and structure simulation. Biotechnology Lett. 30(3): 513-9.

Merino S., Rubires X., Knochel S., Tomas J.M. (1995). Emerging pathogens: Aeromonas spp. Int. J. Food Microbiol. 28:157-168.

Pemberton J.M., Kidd S.P., Schmidt R. (1997). Secreted enzymes of Aeromonas. FEMS Microbiol. Lett. 152:1-10.

Rathore G., Singh V., Kumar G., Swaminathan T.R., Mahanta P.C. (2006). Antibiotic Sensitivity and characterization of Aeromonas hydrophila isolated from fish and water samples. J. Ecophysiol. Occup. Health. 6:41-43.

Siemann, S., Evanoff D.P., Marrone L., Clarke A.J., Viswanatha T., Dmitrienko G.I. (2002). $N$-arylsulfonyl hydrazones as inhibitors of IMP-1 metallo-_lactamase. Antimicrob. Agents Chemother. 46:2450-2457.

Srivastava S.K., Dube D., Tewari N., Dwewedi N., Tripathi R.P., Ramachandran R. (2005). Mycobacterium tuberculosis NAD+ dependent DNA ligase is selectively inhibited by glycosylamins compared with human DNA ligase I. Nucleic Acid Res. 33(22):7090-7101.

Yanez M.A., Catalan V., Apraiz D., Figueras M.J., Martinez-Murcia A.J. (2003). Phylogenetic analysis of members of the genus Aeromonas based on gyrB gene sequences. Int. J. Syst. Evol. Microbiol. 53(3):875-83. 